\title{
A Secondary Field Based $h p$-Finite Element Method for the Simulation of Magnetotelluric Measurements
}

\author{
J. Alvarez-Aramberri ${ }^{\mathrm{a}, \mathrm{b}, \mathrm{c}, *}$, D. Pardo ${ }^{\mathrm{b}, \mathrm{a}, \mathrm{d}}, \mathrm{H}$. Barucq $^{\mathrm{c}, \mathrm{e}}$ \\ ${ }^{a}$ BCAM (Basque Center for Applied Mathematics), Mazarredo 14, 48009, Bilbao, Spain \\ ${ }^{b}$ University of the Basque Country (UPV/EHU), Bilbao, Spain \\ ${ }^{c}$ University of Pau (UPPA), Pau, France \\ ${ }^{d}$ Ikerbasque, Bilbao, Spain \\ ${ }^{e}$ Inria team-project Magique-3D, Pau, France
}

\begin{abstract}
In some geophysical problems, it is sometimes possible to divide the subsurface resistivity distribution as a one dimensional (1D) contribution plus some two dimensional (2D) inhomogeneities. Assuming this scenario, we split the electromagnetic fields into their primary and secondary components, the former corresponding to the $1 \mathrm{D}$ contribution, and the latter to the $2 \mathrm{D}$ inhomogeneities. While the primary field is solved via an analytical solution, for the secondary field we employ a multi-goal oriented self-adaptive $h p$-Finite Element Method (FEM). To truncate the computational domain, we design a Perfectly Matched Layer (PML) that automatically adapts to high-contrast materials that appear in the subsurface and in the air-ground interface. Numerical results illustrate the robustness of the proposed PML and the gains of the secondary field approach, where we obtain results with comparable accuracy than with a full field based formulation but with a much lower computational cost.
\end{abstract}

Keywords: Finite Element Method (FEM), hp-adaptivity, Magnetotelluric Problem, Secondary Field Formulation, Perfectly Matched Layers (PML).

\footnotetext{
* Corresponding author

Email address: julen.alvarez.aramberri@gmail.com (J. Alvarez-Aramberri)
} 


\section{Introduction}

The magnetotelluric (MT) method is a passive exploration technique based on electromagnetic (EM) waves $[1,2,3]$. It aims at estimating the resistivity distribution, and therefore at providing an image of the Earth's subsurface. MT measurements are governed by Maxwell's equations with a surface source located at the ionosphere. In particular, when the materials and the source depend only upon two spatial variables, two independent and uncoupled modes are derived, the so-called Transverse Electric (TE) and Transverse Magnetic (TM) polarizations. The solution to the equations arisen from these two modes can be numerically solved with a $h p$-Finite Element Method (FEM) $[4,5,6,7]$. With those solutions, it is then possible to compute the impedance and/or the apparent resistivity, two suitable physical quantities to perform the inversion.

To correctly capture the complexity of the Earth's subsurface, we employ adaptive grids, which allow to approximate special features of the solution by refining only in specific areas. To build the refined mesh, we employ a goal-oriented adaptive strategy [8], which minimizes the error of a prescribed quantity of interest represented by a linear functional (see [9, 8, 10, 11, 12] for details). The ability of the goal-oriented algorithm to provide accurate solutions in a region of interest in the context of $h p$-FEM has been described in various works $[13,14,15]$. The $h p$-FEM provides exponential convergence rates for elliptic problems with a piecewise analytic solution, whereas $h$ or $p$ versions converge only algebraically. This was proved in 1D by Gui and Babuska [16] and in 2D by Babuska and Gui [17] and Schwab [18].Only the $h p$-FEM is able to combine small elements (needed to capture geometrical details such as thin edges) with high orders of approximation (necessary to decrease the dispersion error for wave propagation problems [19, 20, 21]). Besides, it is robust for singularly perturbed problems, that is, it still performs appropriately when a parameter involved in a given elliptic problem approaches a critical value [18].

In some geophysical applications, as in MTs, the data is acquired at several receivers located at the Earth's surface. It becomes then necessary to obtain accurate results at multiple positions, being this the reason to extend the goal-oriented strategy to a multigoal-oriented one. There exist two possible approaches towards multigoal-oriented adaptivity. The first one consists of using one grid for each goal, as in [22], where the implementation needs to handle multiple grids, which in general may be complicated. The second 
one consists of defining a new quantity of interest that takes into account all goals (see $[23,24]$ ). Based on this second approach, we implement the algorithm proposed by Pardo in [25].

In geophysics in general and in MT in particular, when the subsurface distribution of the resistivity depends upon multiple spatial variables, it is sometimes possible to interpret it as a 1D formation plus some 2D (or 3D) heterogeneities. In this work, we consider a horizontally layered Earth model with 2D heterogeneities. Then, in order to solve the TE and TM modes, we split the electric and magnetic fields into their primary and secondary components. The first corresponds to the fields arisen from some reference conductivity model (1D), while the second arises from the difference between the actual conductivity distribution with respect to the reference model (2D).

Since the 1D solution is known analytically, the main advantage of this approach is that we only need to accurately solve the secondary field variations (the term "secondary field" is also known as "scattered field" in the electrical engineering community), which in general are easier to solve, since they exhibit less variations (smaller gradients) than the primary field. Hence, it is generally possible to employ coarser grids, and hence reduce the computational cost.

Additionally, in MTs the computational domains are usually very large if one models the incident plane wave source. In the secondary field formulation, the source term is not at the ionosphere, but where the inhomogeneities are considered. Since it is not necessary anymore to model the ionosphere source, this allows us to considerably reduce the computational domain. Finally, since we separate the primary from the secondary field, we may obtain additional physical relevant information by analyzing each field (primary and secondary) separately.

The main contribution of this work is then to solve, via the $h p$-FEM, the MT direct problem using the secondary field formulation to simulate MT measurements. The mentioned benefits of this approach will then be notorious in the inversion. On the one hand, there exists the possibility of analyzing 1D and 2D effects separately. On the other hand, since the solution of the inverse problem is based on reiterated solutions of the direct problem, reducing the computational cost of solving the direct problem produces large savings in the computational costs.

Additionally, we provide an automatic technique to truncate the computational domain, a further problem that appears when applying a FEM to unbounded region problems such as MT. Different approaches can be em- 
ployed for this purpose. We employ a Perfectly Matched Layer (PML), which is an exact method at the continuous level, and thus, it matches the highaccuracy delivered by the $h p$-adaptive FEM. The work of Gomez-Revuelto et al. [26] shows the suitability of the utilization of PMLs in this context.

PMLs were proposed by Berenger [27] (1994) in an electromagnetic context as an artificial layer intended to reduce reflections from the boundary of a truncated computational domain. In this method, one has to select the decay profile of the wave into the PML region. This profile needs to ensure that reflections from the boundary are arbitrarily small, which implies that the solution decays arbitrarily fast, creating then a "boundary layer" with strong gradients within the PML region. Thus, while a low decay produces incoming waves reflected from the boundary, an excessive decay requires a very fine grid to approximate the boundary layer. To find an equilibrium between a fast and a slow decay, it is necessary to properly adjust the PML parameters, which is usually tricky since they depend on the problem itself. Moreover, when we have a layered material with high contrasts on the material properties, this selection of the parameters is even more challenging.

Thus, in this work we also provide a method to automatically adjust the PML parameters, even in the most complex scenarios where the material contrast properties among neighboring materials are as high as sixteen orders of magnitude. These type of scenarios often appear in geophysical electromagnetic (EM) applications that involve both, air and ground. We show that the proposed PML produces an appropriate decay of the solution in the air and in the subsurface without introducing spurious reflections, and thus, providing accurate solutions.

The present work is organized as follows. In Section 2 we define the formulation of the problem. Section 3 describes the formulation of the PML and how the parameters are adjust in the Automatically Adapted PML. We derive the secondary field formulation in Section 4 and numerical results based on the MT problem are illustrated in Section 5. Section 6 is devoted to the conclusions.

\section{Formulation of the Method}

MT measurements are governed by the electromagnetic phenomena, which is described by Maxwell's equations. Assuming a time-harmonic dependence 
of the form $e^{j \omega t}$, these equations can be expressed in frequency domain as:

$$
\left\{\begin{array}{lll}
\boldsymbol{\nabla} \times \boldsymbol{E} & =-j \omega \boldsymbol{\mu} \boldsymbol{H}-\boldsymbol{M}^{i m p} & \text { (Faraday), } \\
\boldsymbol{\nabla} \times \boldsymbol{H} & =(\boldsymbol{\sigma}+j \omega \boldsymbol{\varepsilon}) \boldsymbol{E}+\boldsymbol{J}^{i m p} & \text { (Ampère), }
\end{array}\right.
$$

where $\boldsymbol{E}$ and $\boldsymbol{H}$ are the electric and magnetic fields, respectively. These fields are driven by an impressed prescribed electric and magnetic density current sources, $\boldsymbol{J}^{i m p}=\left(0, J_{y}, 0\right)$ and $\boldsymbol{M}^{i m p}=\left(0, M_{y}, 0\right)$, respectively. We emphasize that, as explained in [28], magnetic impressed currents are only mathematical symbols utilized to represent sources. $j$ is the imaginary unit, $\omega$ is the angular frequency, and $\boldsymbol{\sigma}$ stands for the conductivity of the media. We assume that

$$
\boldsymbol{\sigma}=\left(\begin{array}{ccc}
\sigma & 0 & 0 \\
0 & \sigma & 0 \\
0 & 0 & \sigma
\end{array}\right), \boldsymbol{\varepsilon}=\left(\begin{array}{ccc}
\varepsilon & 0 & 0 \\
0 & \varepsilon & 0 \\
0 & 0 & \varepsilon
\end{array}\right), \boldsymbol{\mu}=\left(\begin{array}{ccc}
\mu & 0 & 0 \\
0 & \mu & 0 \\
0 & 0 & \mu
\end{array}\right)
$$

where the electrical permitivity $\varepsilon$ and the magnetic permeability $\mu$ are assumed to be that of the vacuum ( $\varepsilon_{0}$ and $\mu_{0}$ respectively) and $\sigma(x, y, z)$ to be piecewise constant, non-negative, and bounded above.

\subsection{Transverse Electric (TE) and Transverse Magnetic (TM) Modes}

Pre-multiplying both sides of Faraday's Law by $\boldsymbol{\mu}^{-1}$, applying the curl, and using Ampère's Law, we obtain the reduced wave equation,

$$
\boldsymbol{\nabla} \times\left(\boldsymbol{\mu}^{-1} \nabla \times \boldsymbol{E}\right)-\boldsymbol{k}^{2} \boldsymbol{E}=-j \omega \boldsymbol{J}^{i m p}-\nabla \times\left(\boldsymbol{\mu}^{-1} \boldsymbol{M}^{i m p}\right),
$$

where $\boldsymbol{k}^{2}=\omega^{2} \boldsymbol{\varepsilon}-j \omega \boldsymbol{\sigma}$. A similar equation is obtained in terms of the magnetic field by multiplying both sides of Ampère's Law by $\hat{\boldsymbol{\sigma}}^{-1}=(\boldsymbol{\sigma}+$ $j \omega \varepsilon)^{-1}$ and applying the curl to Faraday's Law

$$
\boldsymbol{\nabla} \times\left(\hat{\boldsymbol{\sigma}}^{-1} \boldsymbol{\nabla} \times \boldsymbol{H}\right)+j \omega \boldsymbol{\mu} \boldsymbol{H}=-\boldsymbol{M}^{i m p}+\boldsymbol{\nabla} \times\left(\hat{\boldsymbol{\sigma}}^{-1} \boldsymbol{J}^{i m p}\right) .
$$

When the materials and the source depend only upon two spatial variables $(x, z)$, then $\partial / \partial y=0$ and two independent and uncoupled modes are derived from Maxwell's equations. The uncoupled TE mode involves $\left(E_{y}, H_{x}, H_{z}\right)$ field components, while TM only considers $\left(H_{y}, E_{x}, E_{z}\right)$. Our aim is to find the $y$ component of the electric and magnetic fields $E_{y}(x, z), H_{y}(x, z) \in$ $H^{1}(\Omega)$ that satisfy the BCs and equations (3), and (4), respectively. 
To derive the variational formulation, we first define the $L^{2}$-inner product of two possible complex and vector valued functions $\boldsymbol{g}_{\mathbf{1}}$ and $\boldsymbol{g}_{\mathbf{2}}$ as:

$$
\left\langle\boldsymbol{g}_{\mathbf{1}}, \boldsymbol{g}_{\mathbf{2}}\right\rangle_{L^{2}(\Omega)}=\int_{\Omega} \boldsymbol{g}_{\mathbf{1}}{ }^{*} \boldsymbol{g}_{\mathbf{2}} d \Omega
$$

where $\boldsymbol{g}^{*}$ denotes the adjoint (transpose of the complex conjugate) of $\boldsymbol{g}$.

\subsubsection{TE Variational Formulation}

To obtain the corresponding variational formulation, we pre-multiply (3) by the complex conjugate of a test function $F \in V(\Omega)$, where $V(\Omega)=$ $H_{\Gamma_{D}}^{1}(\Omega)=\left\{F \in L^{2}(\Omega):\left.F\right|_{\Gamma_{D}}=0, \nabla F \in \boldsymbol{L}^{2}(\Omega)\right\}$ is the space of admissible test functions. Then, we integrate by parts and we incorporate the homogeneous Dirichlet BC (the ones considered in the present work) over $\Gamma_{D}=\partial \Omega$. Thus, we obtain:

$$
\left\{\begin{array}{l}
\text { Find } \quad E_{y} \in V(\Omega), \quad \text { such that: } \\
\left\langle\nabla F, \mu^{-1} \nabla E_{y}\right\rangle_{L^{2}(\Omega)}-\left\langle F, k^{2} E_{y}\right\rangle_{L^{2}(\Omega)}=-j \omega\left\langle F, J_{y}^{i m p}\right\rangle_{L^{2}(\Omega)} \quad \forall F \in V(\Omega),
\end{array}\right.
$$

\subsubsection{TM Variational Formulation}

In a similar way, from (4) we obtain the corresponding variational formulation for the magnetic field

$$
\left\{\begin{array}{l}
\text { Find } \quad H_{y} \in V(\Omega), \quad \text { such that: } \\
\left\langle\nabla F, \hat{\sigma}^{-1} \nabla H_{y}\right\rangle_{L^{2}(\Omega)}+j \omega\left\langle F, \mu H_{y}\right\rangle_{L^{2}(\Omega)}=-\left\langle F, M_{y}^{i m p}\right\rangle_{L^{2}(\Omega)} \quad \forall F \in V(\Omega),
\end{array}\right.
$$

We employ an $h p$-Finite Element Method [4] to solve both problems (6) and (7). The objective of the traditional goal-oriented method is to construct an optimal $h p$-grid in the sense that it minimizes the problem size needed to achieve a given tolerance error for a given quantity of interest (solution at the receiver) $L^{i}(u)$, being $u$ either $E_{y}$ or $H_{y}$. This quantity is a linear and continuous functional $[14,15]$ in $u$ associated to the $i$-th receiver and defined as:

$$
L^{i}(u)=\frac{1}{\left|\Omega_{R^{i}}\right|} \int_{\Omega_{R^{i}}} u d \Omega
$$

where $\Omega_{R^{i}}$ is the domain occupied by the $i$-th receiver. 
Since we have more than one receiver, we need to properly compute several quantities of interest. Therefore, we employ a multigoal-oriented strategy, proposed in [25], where a new linear quantity of interest that takes into account all receivers is employed.

From the solution to the variational problems, we compute the impedance and/or the apparent resistivity, which are two post-processed transfer functions that are typically used during inversion in MT problems. The impedance $\mathcal{Z}$ is defined as

$$
\mathcal{Z}_{T E}^{i}=\mathcal{Z}_{y x}^{i}=\frac{L^{i}\left(E_{y}\right)}{L^{i}\left(H_{x}\right)}, \quad \mathcal{Z}_{T M}^{i}=\mathcal{Z}_{x y}^{i}=\frac{L^{i}\left(E_{x}\right)}{L^{i}\left(H_{y}\right)},
$$

where $H_{x}$ and $E_{y}$ are obtained from Maxwell's equations as

$$
H_{x}=\frac{1}{j \omega \mu} \frac{\partial E_{y}}{\partial z}, \quad E_{x}=-\frac{1}{\sigma+j \omega \varepsilon} \frac{\partial H_{y}}{\partial z} .
$$

The apparent resistivity $\rho^{a p p}$ is defined as

$$
\rho_{m n}^{a p p}=\frac{\left|\mathcal{Z}_{m n}\right|^{2}}{\omega \mu} .
$$

For the sake of simplicity in the notation, we omit the $y$ subscript from $E_{y}$, $H_{y}, J_{y}^{i m p}$, and $M_{y}^{i m p}$ from now on.

\section{Truncation of the Domain}

When applying a FEM to unbounded region problems such as MT, the computational domain must be truncated. We employ PMLs for this purpose, and we follow the interpretation introduced by Teixera and Chew in $[29,30]$, where they consider a PML as an analytic continuation of the governing equations into the complex plane (see also [31]).

PMLs transform propagating and evanescent waves into exponentially fast decaying evanescent waves. Since waves are strongly attenuated inside the PML region, the bounded computational domain can be limited by a surface on which one can set perfectly reflecting BCs (in our case, we set homogeneous Dirichlet BCs). Indeed, any reflected wave is so much absorbed inside the layer that it does not pollute the solution inside the domain of study. Then, the selected BCs for both problems imply that the tangential component of the fields are zero on the outer part of the boundary. For a recent review of the state of the art of this truncation technique, see [32] and [33]. 


\subsection{PML Definition}

Let the Cartesian coordinate system $\boldsymbol{x}=(x, z)$ be the reference system of coordinates in a 2D scenario, where for simplicity, we select the vertical coordinate $z$ as the direction perpendicular to the ground-air interface. Given an arbitrary complex system of coordinates $\boldsymbol{\zeta}=\left(\zeta_{1}, \zeta_{2}\right)$, we define our change of coordinates by $\boldsymbol{x}=\boldsymbol{\psi}(\boldsymbol{\zeta})$ and we denote the Jacobian matrix and its determinant by $\mathcal{J}$ and $\operatorname{det}(\mathcal{J})$. The change of coordinates is assumed to be represented by an injective differentiable function with continuous partial derivatives and nonzero determinant at any point.

We define a one dimensional change of variables in the positive direction of the $i$-th coordinate as

$$
\zeta_{x_{i}}\left(x_{i}\right)=\int_{0}^{x_{i}} h(\eta) d \eta, \text { for } i=1,2, x_{1}=x, x_{2}=z,
$$

where $h(\cdot)$ is a possibly complex valued function to be determined in section 3.3. The case corresponding to the negative direction can be defined analogously. The Jacobian is given by $[\mathcal{J}]_{i, j}=\left[\frac{\partial \zeta_{i}}{\partial x_{j}}\right]_{i, j}$, for $i, j=1,2$. Thus, it is expressed as

$$
\mathcal{J}=\left(\begin{array}{cc}
h(x) & 0 \\
0 & h(z)
\end{array}\right), \quad \text { where } \quad \operatorname{det}(\mathcal{J})=h(x) h(z)
$$

denotes the determinant of the Jacobian.

With this particular change of coordinates, the Jacobian is diagonal. However, we derive herein the variational formulation for a general, non orthogonal change of variables. This is useful for other purposes, e.g. development of non-orthogonal Fourier FEMs in certain geometries (see [34, 35]).

\subsection{Variational Formulation in an Arbitrary System of Coordinates}

We define the change of coordinates $\widetilde{E}:=E \circ \boldsymbol{\psi}=\widetilde{E}(\boldsymbol{\zeta}), \widetilde{F}:=F \circ$ $\boldsymbol{\psi}=\widetilde{F}(\boldsymbol{\zeta})$, and $\widetilde{J}^{i m p}:=J^{i m p} \circ \boldsymbol{\psi}=J^{i m p}(\boldsymbol{\zeta})$. Using Einstein's summation convention, according to the chain rule, denoting with the upper bar the complex conjugate, and taking into account that if $f \in \mathrm{C}^{1}(\Omega)$, then for all $i$

$$
\frac{\overline{\partial f}}{\partial \zeta_{i}}=\frac{\partial \bar{f}}{\partial \bar{\zeta}_{i}}, \quad \frac{\overline{\partial f}}{\partial \bar{\zeta}_{i}}=\frac{\partial \bar{f}}{\partial \zeta_{i}}
$$


we obtain that

$$
\begin{aligned}
\nabla^{\zeta} \widetilde{E} & =\frac{\partial \widetilde{E}}{\partial x_{i}} \frac{\partial x_{i}}{\partial \zeta_{n}} \boldsymbol{e}_{x_{n}}=\left(\mathcal{J}^{-1}\right)^{T} \nabla E \\
\nabla^{\bar{\zeta}} \widetilde{E} & =\frac{\partial \widetilde{E}}{\partial x_{i}} \frac{\partial x_{i}}{\partial \bar{\zeta}_{n}} \boldsymbol{e}_{x_{n}}=\left(\mathcal{J}^{-1}\right)^{*} \nabla E
\end{aligned}
$$

Therefore, multiplying (3) by the complex conjugate of a test function $\widetilde{F}$, integrating by parts, and incorporating the homogeneous Dirichlet $\mathrm{BC}$ over $\widetilde{\Gamma}_{D}$, we obtain:

$$
\begin{aligned}
\left\langle\nabla^{\bar{\zeta}} \widetilde{F}, \widetilde{\mu}^{-1} \nabla^{\zeta} \widetilde{E}\right\rangle_{L^{2}(\widetilde{\Omega})} & =\left\langle\left(\mathcal{J}^{-1}\right)^{*} \nabla F, \mu^{-1}\left(\mathcal{J}^{-1}\right)^{T} \nabla E \operatorname{det}(\mathcal{J})\right\rangle_{L^{2}(\Omega)}= \\
& =\left\langle\nabla F, \mathcal{J}^{-1} \mu^{-1}\left(\mathcal{J}^{-1}\right)^{T} \nabla E \operatorname{det}(\mathcal{J})\right\rangle_{L^{2}(\Omega)}, \\
\left\langle\widetilde{F}, \widetilde{k}^{2} \widetilde{E}\right\rangle_{L^{2}(\widetilde{\Omega})} & =\left\langle F, k^{2} E \operatorname{det}(\mathcal{J})\right\rangle_{L^{2}(\Omega)}, \\
\left\langle\widetilde{F}, \widetilde{J}^{i m p}\right\rangle_{L^{2}(\widetilde{\Omega})} & =\left\langle F, J^{i m p} \operatorname{det}(\mathcal{J})\right\rangle_{L^{2}(\Omega)},
\end{aligned}
$$

where $\widetilde{\mu}:=\mu \circ \boldsymbol{\psi}, \widetilde{k}:=k \circ \boldsymbol{\psi}, \widetilde{\Omega}:=\Omega \circ \boldsymbol{\psi}$, and $\widetilde{\Gamma}_{D}:=\Gamma_{D} \circ \boldsymbol{\psi}$.

Following the ideas of [36] concerning the inclusion of metric-dependent variables within material coefficients, we define the following functions:

$$
\begin{aligned}
\boldsymbol{\mu}_{N E W}^{T E}=\mathcal{J}^{T} \mu \mathcal{J} \frac{1}{\operatorname{det}(\mathcal{J})}=\left(\begin{array}{cc}
\mu \frac{h(x)}{h(z)} & 0 \\
0 & \mu \frac{h(z)}{h(x)}
\end{array}\right), \\
k_{N E W}^{2}=k^{2} \operatorname{det}(\mathcal{J})=k^{2} h(x) h(z), \\
J_{N E W}^{i m p}=J^{i m p} \operatorname{det}(\mathcal{J})=J^{i m p} h(x) h(z) .
\end{aligned}
$$

The new source and new material tensors incorporate the information about the change of coordinates. Thus, the variational formulation can be expressed in terms of an arbitrary system of coordinates by simply considering the new source and materials. The new variational formulation for the electric field 
in the new system of coordinates is then given by

$$
\left\{\begin{aligned}
& \text { Find } \quad E \in \tilde{V}(\Omega), \quad \text { such that: } \\
& \begin{array}{rl}
\left\langle\nabla F,\left(\boldsymbol{\mu}_{N E W}^{T E}\right)^{-1} \nabla E\right\rangle_{L^{2}(\Omega)} & -\left\langle F, k_{N E W}^{2} E\right\rangle_{L^{2}(\Omega)}= \\
& -j \omega\left\langle F, J_{N E W}^{i m p}\right\rangle_{L^{2}(\Omega)}, \quad \forall F \in \tilde{V}(\Omega),
\end{array}
\end{aligned}\right.
$$

and an analogous procedure for the magnetic field yields

$$
\left\{\begin{aligned}
\text { Find } H \in \tilde{V}(\Omega), & \text { such that: } \\
\left\langle\nabla F, \hat{\boldsymbol{\sigma}}_{N E W}^{-1} \boldsymbol{\nabla} H\right\rangle_{L^{2}(\Omega)}+j \omega & \left\langle F, \mu_{N E W}^{T M} H\right\rangle_{L^{2}(\Omega)}= \\
& -\left\langle F, M_{N E W}^{i m p}\right\rangle_{L^{2}(\Omega)}, \quad \forall F \in \tilde{V}(\Omega),
\end{aligned}\right.
$$

where $\tilde{V}(\Omega)=\widetilde{H}_{\Gamma_{D}}^{1}(\Omega)=\left\{E \in L^{2}(\Omega):\left.E\right|_{\Gamma_{D}}=0,\left(\mathcal{J}^{-1}\right)^{T} \nabla E \in \boldsymbol{L}^{2}(\Omega)\right\}$ in both cases, and new materials and sources for the magnetic field are now given by:

$$
\begin{aligned}
& \hat{\boldsymbol{\sigma}}_{N E W}=\mathcal{J}^{T} \hat{\sigma} \mathcal{J} \frac{1}{\operatorname{det}(\mathcal{J})}=\left(\begin{array}{cc}
\hat{\sigma} \frac{h(x)}{h(z)} & 0 \\
0 & \hat{\sigma} \frac{h(z)}{h(x)}
\end{array}\right), \\
& \mu_{N E W}^{T M}=\mu \operatorname{det}(\mathcal{J})=\mu h(x) h(z), \\
& M_{N E W}^{i m p}=M^{i m p} \operatorname{det}(\mathcal{J})=M^{i m p} h(x) h(z) .
\end{aligned}
$$

\subsection{Automatic Adjustment of PML Parameters}

Given the general change of coordinates (12), we select

$$
h\left(x_{i}\right)= \begin{cases}1, & x_{i}<x_{i}^{a} \\ \phi_{i} \in \mathbb{C}, & x_{i} \in\left[x_{i}^{a}, x_{i}^{b}\right),\end{cases}
$$

where $\left[x_{i}^{a}, x_{i}^{b}\right)$ defines the position of the PML in the positive direction of the $i$-th coordinate.

Far away from the source, the solution can be assumed to be a sum of plane waves. Thus, it may be decomposed into functions of the form $e^{-j \beta \boldsymbol{x} \cdot \boldsymbol{v}}$ (see [37]), where $\beta=\sqrt{\omega^{2} \mu \epsilon-j \omega \mu \sigma}$ is the wavenumber and $\boldsymbol{v}$ is an unit vector that indicates the direction of propagation of the wave. 
When implementing a PML, one has to select the decay profile of the wave into the PML region, which is usually a tricky task due to the dependence on the problem itself. To find an equilibrium between a fast and a slow decay, it is neecessary to properly adjust the PML parameters. Moreover, for layered formations with high contrasts on the material properties, this selection of the parameters is even more challenging.

The worst scenario, the one in which the wave travels the shortest distance, corresponds to a plane wave traveling perpendicularly to the PML towards the positive direction of the $x_{i}$ variable (an analogous analysis can be performed for the negative direction). Then, the solution in the PML region for the new variable is given by

$$
E\left(\zeta\left(x_{i}\right)\right)=e^{-j \beta \zeta\left(x_{i}\right)}=e^{-j \beta \phi_{i} x_{i}} .
$$

Our aim is to make the solution vanish in the PML region without introducing reflections. Therefore, we define the decay factor $\alpha$, which measures the decay of the solution from $x_{i}^{a}$ to $x_{i}^{b}$, as

$$
\alpha:=\frac{E\left(x_{i}^{b}\right)}{E\left(x_{i}^{a}\right)}=e^{-j \beta \phi_{i}\left(x_{i}^{b}-x_{i}^{a}\right)} .
$$

Then, we select

$$
\phi_{i}=j \frac{\log (\alpha)}{\beta\left(x_{i}^{b}-x_{i}^{a}\right)} .
$$

Remark. With this formula we obtain the final definition of the Automatically Adapted PML. It adjusts the parameters without further user interaction, even if the material properties change abruptly. This is especially important for our MT application, since we have layers with highly varying conductivities. The most prominent example of this occurs on the air-Earth interface. The only user interaction is to define the desired decay into the PML region, i.e., $\alpha$. Then, $\phi_{i}$ is automatically computed guaranteeing a minimum decay in the outer part of the PML according to the selected $\alpha$.

\section{Secondary Field Formulation}

We consider the reference conductivity model as a 1D layered media with a known analytical solution (see [38] for instance), and we denote it by $\boldsymbol{\sigma}^{P}$. Let $\Omega_{2}$ be the domain where the $2 \mathrm{D}$ inhomogeneities are located while $\Omega_{1}$ corresponds to the remaining part (see Figure 1). Defining $\boldsymbol{\sigma}^{S}=\boldsymbol{\sigma}-\boldsymbol{\sigma}^{P}$, then 


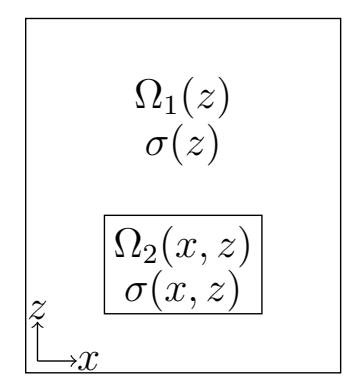

Figure 1: Illustration of the computational subdomains $\Omega_{1}$ and $\Omega_{2}$ and the material properties inside each subdomain. $\sigma$ only depends on $z$ in $\Omega_{1}$, whereas on $x$ and $z$ in $\Omega_{2}$.

$\boldsymbol{\sigma}^{S}$ is equal to zero outside $\Omega_{2}$. If we denote the solution to the primary field by $\left(\boldsymbol{E}^{P}, \boldsymbol{H}^{P}\right)$, then the secondary field $\left(\boldsymbol{E}^{S}, \boldsymbol{H}^{S}\right)$ is defined as the difference between the total and the primary fields as:

$$
\boldsymbol{E}^{S}=\boldsymbol{E}-\boldsymbol{E}^{P}, \quad \boldsymbol{H}^{S}=\boldsymbol{H}-\boldsymbol{H}^{P} .
$$

Under these considerations, the equations for the electric (3) and magnetic (4) fields become:

$$
\begin{gathered}
\boldsymbol{\nabla} \times\left(\boldsymbol{\mu}^{-1} \boldsymbol{\nabla} \times \boldsymbol{E}^{S}\right)-\boldsymbol{k}^{2} \boldsymbol{E}^{S}=-j \omega \boldsymbol{\sigma}^{S} \boldsymbol{E}^{P}, \\
\boldsymbol{\nabla} \times\left(\hat{\boldsymbol{\sigma}}^{-1} \boldsymbol{\nabla} \times \boldsymbol{H}^{S}\right)+j \omega \boldsymbol{\mu} \boldsymbol{H}^{S}=-\boldsymbol{\nabla} \times\left(\tilde{\boldsymbol{\sigma}}^{-1} \boldsymbol{\nabla} \times \boldsymbol{H}^{P}\right)
\end{gathered}
$$

where $\tilde{\boldsymbol{\sigma}}^{-1}=\hat{\boldsymbol{\sigma}}^{-1}-\left(\hat{\boldsymbol{\sigma}}^{P}\right)^{-1}$.

These equations yield a similar weak formulation to the one derived in the previous section. The main modification is on the right hand side. This implies that after incorporating the PML, the new materials are also computed in the same way. In particular, for a 2D model Earth with a PML, we obtain that the variational problems are stated as

$$
\left\{\begin{array}{l}
\text { Find } E^{S} \in \tilde{V}(\Omega), \quad \text { such that: } \\
\begin{array}{rl}
\left\langle\nabla F,\left(\boldsymbol{\mu}_{N E W}^{T E}\right)^{-1} \nabla E^{S}\right\rangle_{L^{2}(\Omega)} & -\left\langle F, k_{N E W}^{2} E^{S}\right\rangle_{L^{2}(\Omega)}= \\
& -j \omega\left\langle F, \sigma_{N E W}^{S} E^{P}\right\rangle_{L^{2}(\Omega)} \quad \forall F \in \tilde{V}(\Omega),
\end{array}
\end{array}\right.
$$


for the electric field, and

$$
\left\{\begin{array}{c}
\text { Find } H^{S} \in \tilde{V}(\Omega), \quad \text { such that: } \\
\begin{array}{rl}
\left\langle\nabla F, \hat{\boldsymbol{\sigma}}_{N E W}^{-1} \boldsymbol{\nabla} H^{S}\right\rangle_{L^{2}(\Omega)}+j \omega & \left\langle F, \mu_{N E W}^{T M} H^{S}\right\rangle_{L^{2}(\Omega)}= \\
& -\left\langle\boldsymbol{\nabla} F, \tilde{\boldsymbol{\sigma}}_{N E W}^{-1} \boldsymbol{\nabla} H^{P}\right\rangle_{L^{2}(\Omega)}, \quad \forall F \in \tilde{V}(\Omega),
\end{array}
\end{array}\right.
$$

for the magnetic field. $\tilde{\boldsymbol{\sigma}}_{N E W}$ is then given by

$$
\tilde{\boldsymbol{\sigma}}_{N E W}=\left(\begin{array}{cc}
\tilde{\sigma} \frac{h(x)}{h(z)} & 0 \\
0 & \tilde{\sigma} \frac{h(z)}{h(x)}
\end{array}\right)
$$

and in both cases we have that $\widetilde{V}(\Omega)=\widetilde{H}_{\Gamma_{D}}^{1}(\Omega)=\left\{F \in L^{2}(\Omega):\left.F\right|_{\Gamma_{D}}=\right.$ $\left.0,\left(\mathcal{J}^{-1}\right)^{T} \nabla F \in \boldsymbol{L}^{2}(\Omega)\right\}$.

Because of the linearity of $L^{i}$, we compute the quantities of interest as:

$$
L^{i}(\boldsymbol{E})=L^{i}\left(\boldsymbol{E}^{P}\right)+L^{i}\left(\boldsymbol{E}^{S}\right),
$$

and

$$
L^{i}(\boldsymbol{H})=L^{i}\left(\boldsymbol{H}^{P}\right)+L^{i}\left(\boldsymbol{H}^{S}\right) .
$$

Notice that the use of a secondary field formulation can be straightforwardly extended to a 3D problem when considering 3D inhomogeneities.

\section{Numerical Results}

In this section we study the impact of the automatically adjusted PML into our MT model problem, and we illustrate the savings obtained from using the secondary field formulation as opposed to the full field formulation. To limit the paper size and for simplicity, in this section we display results obtained with the TE mode only.

\subsection{Model Problem}

We assume a horizontally layered Earth model with some 2D inhomogeneities. We model the source as an infinitely long (in $x$ and $y$ directions) rectangular surface located at the ionosphere. This allows us to treat the 


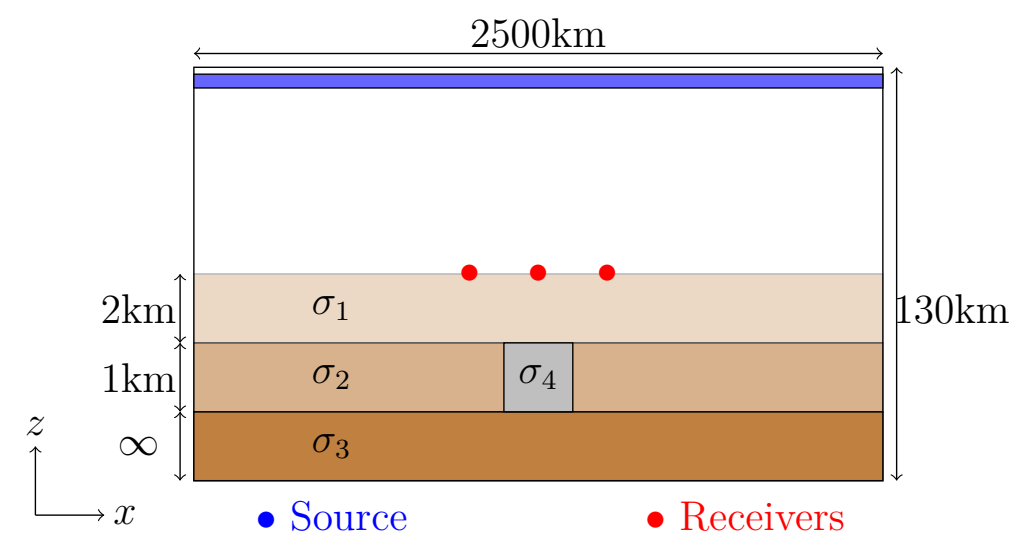

Figure 2: 2D MT problem. Blue rectangle: natural source. Red circles: receivers.

electromagnetic fields as plane waves that propagate in the vertical direction towards the Earth's surface [2].

The physical problem is illustrated in Figure 2. The computational domain in consideration, of $2500 \times 130 \mathrm{~km}^{2}$, consists of air and a layered media (eventually with a target region) for modelling the subsurface formation. The horizontal dimension corresponds to the $x$ spatial variable (with zero at the center), and the vertical to the $z$ (with zero on the surface). The physical domain is truncated with a PML and a Dirichlet homogeneous BCs imposed at the PML's outer part. The relative permittivity and permeability are the same in all materials, and equal to one, while we set different values for the conductivity $\left(\sigma_{1}, \ldots, \sigma_{4}\right)$ for the numerical results. The receivers are located at the Earth's surface and are represented with red circles. The source, located at the ionosphere, is represented with a dark blue rectangle. When the media is only $1 \mathrm{D}$ dependent (invariant in $x$ ), the solution along the surface is constant ( $x$ and $y$ independent), and therefore, it is sufficient to consider only one receiver. However, when 2D inhomogeneities are considered, we are interested in having the solution at different horizontal positions.

Figure 3 illustrates the computational domain when the secondary field formulation is considered. In this case, the source is located at the $2 \mathrm{D}$ inhomogeneities. For both domains (full and smaller one), we select the forcing term $J^{i m p}=1$.

For the 1D problem, we implement the exact solution proposed by Chew [38], and for the numerical calculations we employ the multigoal-oriented $h p$ - 


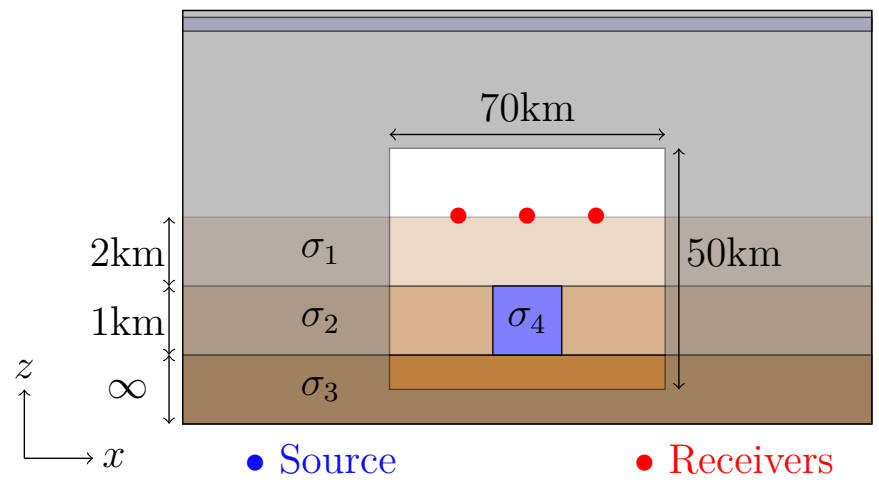

Figure 3: 2D MT Problem for the Secondary Field. The light area represents the new (smaller) computational domain considered for the secondary field formulation. Blue square: source region for the secondary field problem. Red circles: receivers.

FEM $[25,6]$, which ensures accurate solutions at all receivers.

\subsection{Validation Results}

We first ensure that our automatically adapted PML provides accurate solutions when using the $h p$-FEM with the full formulation (without the secondary field formulation) approach.

We consider a $5 \mathrm{~km}$ thick PML. A geometric scaling of the initial mesh in each direction of the PML region is depicted in Figure 4. The number of elements into the PML region typically represents approximately the $15 \%$ of the total amount of elements. We define a decay factor of $\alpha=10^{-5}$, ensuring

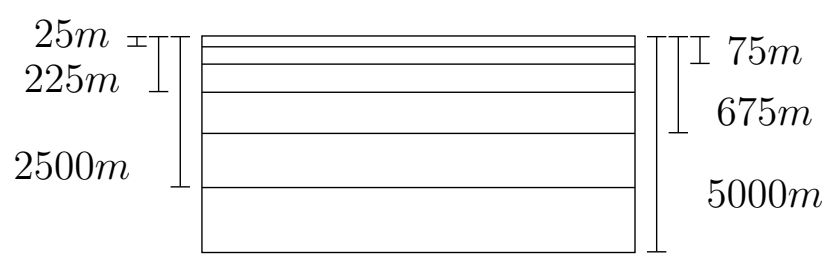

Figure 4: Layers of the FEM mesh in an arbitrary direction into the PML region.

that the wave decays sufficiently fast when arriving to the boundary of the domain.

We consider four 1D model problems with analytical solution, as described in Table 1. In Figure 5 we display the relative error at different 


\begin{tabular}{cccc}
\hline & $\sigma_{1}$ & $\sigma_{2}$ & $\sigma_{3}$ \\
\hline Model 1 & 1 & 1 & 1 \\
Model 2 & 1 & $1 / 10$ & $1 / 3$ \\
Model 3 & 1 & $1 / 10$ & $1 / 10$ \\
Model 4 & 1 & $1 / 100$ & $1 / 3$ \\
\hline
\end{tabular}

Table 1: Different models for the formation of the subsurface. Conductivities are given in $\mathrm{S} / \mathrm{m}$.

frequencies between the numerical $h p$-FEM solution and the exact solution. We obtain relative errors below 1.5\%, a superb accuracy for these type of simulations.

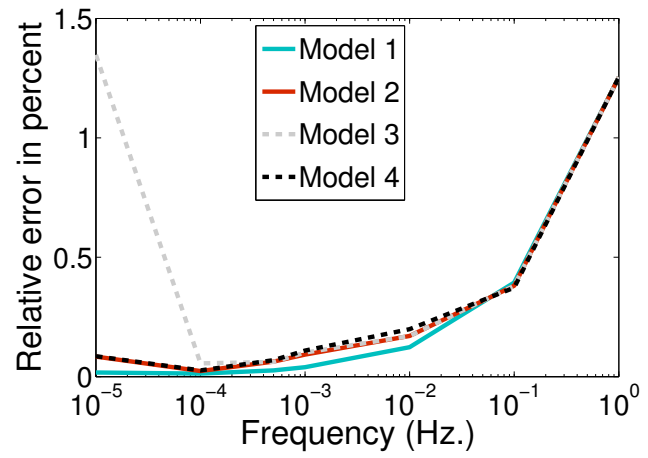

Figure 5: Relative error between the exact and numerical solutions for different subsurface formations against frequency.

To study the behavior of the solution into the PML region, we consider Model 4 with frequency equal to $10^{-4} \mathrm{~Hz}$ and we display the logarithm of the module of the impedance along all sides of the computational domain. Thus, we represent $\log \left(\left|\mathcal{Z}_{T E}\right|\right)$ in Figure 6. We appreciate that the PML behaves properly everywhere, with a smooth decay for the solution and without introducing numerical reflections even in the areas with high contrast between material properties. Panels (a) and (b) correspond to the intersection between air and ground. There, the contrast between resistivities is about sixteen orders of magnitude and even in this scenario, the decay seems to be superb. 

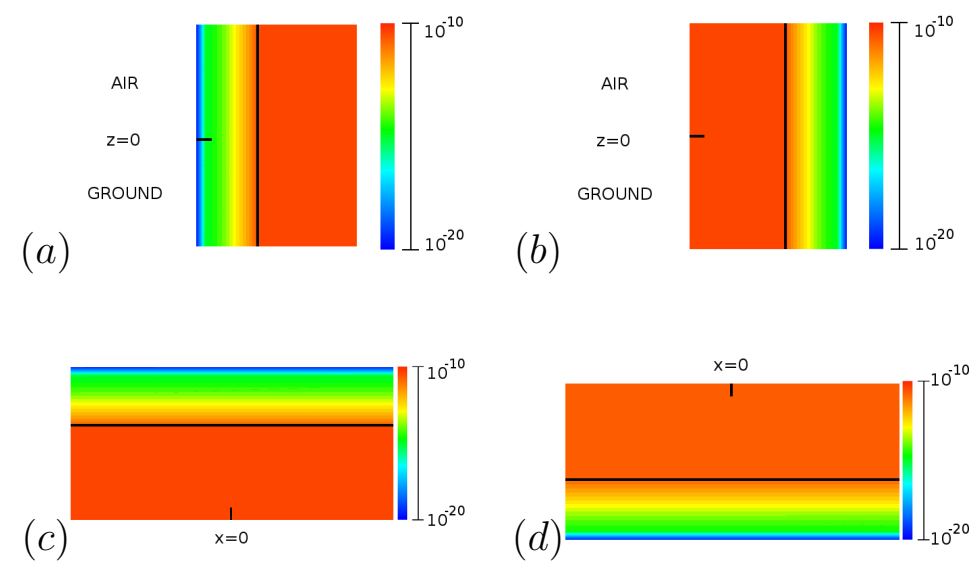

Figure 6: $\log \left(\left|\mathcal{Z}_{T E}\right|\right)$ corresponding to Model 4, with a $5 \mathrm{~km}$ thick PML and $\alpha=$ $10^{-5}$. Panel (a) corresponds to the left side of the domain. (b), (c), and (d) correspond to the right, top and bottom parts of the domain, respectively. The black line indicates the region where the PML starts.

\subsection{Secondary Field Formulation}

We consider now a $2 \mathrm{D}$ scenario with the following conductivity distribution: $\sigma_{1}=1 / 3, \sigma_{2}=1 / 2, \sigma_{3}=1 / 4, \sigma_{4}=1 / 200 \mathrm{~S} / \mathrm{m}$. The most sensitive frequency to the target area, that is, the frequency at which the presence of the target affects most the measurements at the receivers, corresponds to $0.05 \mathrm{~Hz}$. Figure 7 shows the final grids after executing the multi-goal oriented adaptivity for the full formulation (left) and for the secondary field based problem (right) at this frequency. The left panel shows a zoom of the final grid with the origin of coordinates at the center. The size of the represented domain is of $40 \times 70 \mathrm{~km}^{2}$. The grid in the right panel is the complete grid for the secondary field problem $(50 \times 70) \mathrm{km}^{2}$.

Figure 8 displays the relative errors in the apparent resistivity between the full field and secondary field solutions. There, positions 1 to 4 correspond to measurements obtained at $0,4,8$ and $20 \mathrm{~km}$ from the center of the domain, respectively. Due to the low errors observed in Figure 8, we conclude that both approaches provide analogous results. However, the number of unknowns needed to achieve these small errors are not the same.

We now consider the same model with the same frequency of $0.05 \mathrm{~Hz}$. We compute an overkill solution with a much finer grid obtained after performing 

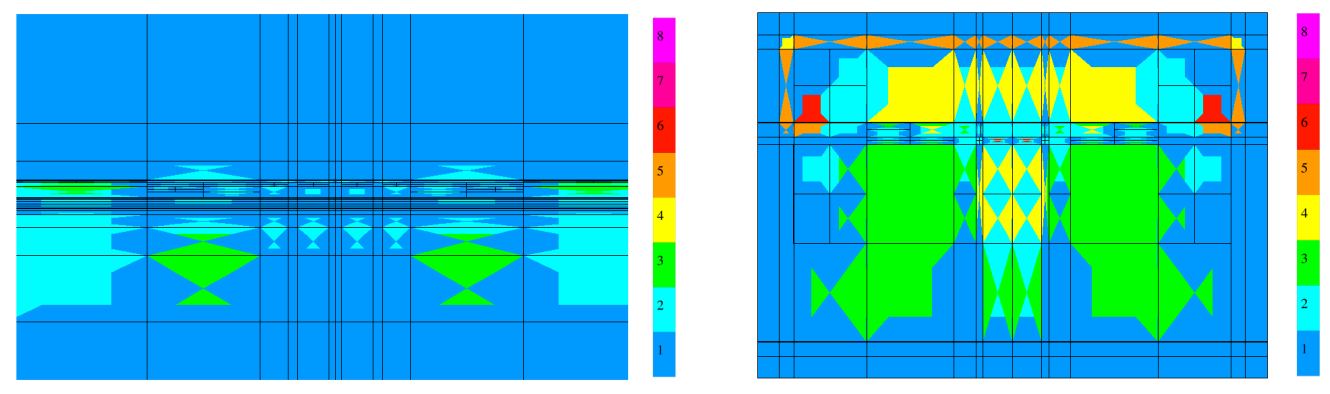

Figure 7: Final multi-goal oriented $h p$-grids. Different colors indicate different values of $p$. Left: full formulation based problem (zoom). Right: secondary field based problem.

adaptivity. We use it to estimate the relative errors corresponding to the secondary field and full formulations after several $h$ and/or $p$ global refinements. Figure 9 displays the results of these computations for the receiver located at the center of the domain. We appreciate that, for instance, to achive a (small) relative error of $0.1 \%$, one only requires around 7000 unknowns with the secondary field formulation, while to solve the full formulation problem with the same accuracy, we need around 17000 unknowns. Therefore, with the first approach we only need approximately $40 \%$ of the unknowns.

\section{Conclusions}

The multi-goal oriented $h p$-FEM provides accurate solutions for the MT problem at different receivers simultaneously. We show that by employing the secondary field approach, we obtain significant benefits in comparison with directly using the full field formulation: we can obtain additional physical relevant information by analyzing each field (primary and secondary) separately, and furthermore, this is obtained employing a significantly lower number of unknowns. Since the solution of the inverse problem is based on iterated solutions of the direct problem, reducing the computational cost of solving the direct problem induces high savings in the inversion process.

We also provide a method to automatically truncate the computational domain employing PMLs. To find an equilibrium between a fast and a slow decay on the PML region is usually tricky. It depends on the problem itself and it is even more complicated when there exists high contrasts on adjacent 


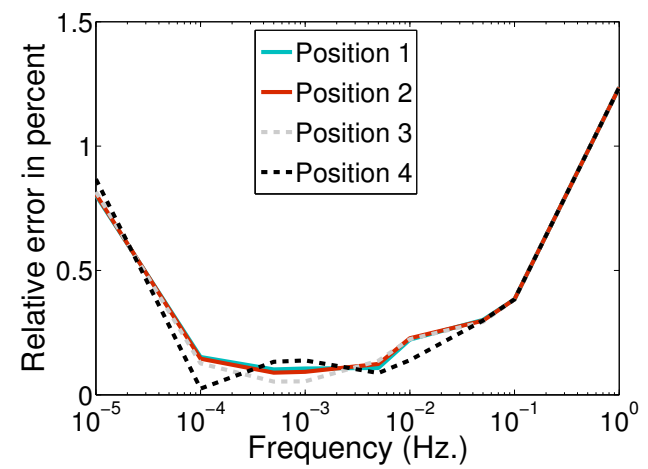

Figure 8: Comparison between the results when using the full formulation and the secondary field formulation.

material properties. We have shown that in these complicated scenarios, the Automatically Adapted PML provides an adequate decay, not so fast to require a too fine grid and not so slow to introduce artificial reflections. Since the choice of PML parameters is automatic, the proposed approach is also suitable for inverse problems.

Even if the reduction of the computational cost is itself beneficial, the main advantage of solving the inverse problem with this approach consists on the fact that it allows for separate analysis of $1 \mathrm{D}$ and $2 \mathrm{D}$ effects. This will be analyzed in future research.

\section{Acknowledgments}

Julen Alvarez-Aramberri and David Pardo were partially funded by the Project of the Spanish Ministry of Economy and Competitiveness with reference MTM2013-40824-P, the BCAM "Severo Ochoa" accreditation of excellence SEV-2013-0323, the CYTED 2011 project 712RT0449, and the Basque Government through the BERC 2014-2017 program and the Consolidated Research Group Grant IT649-13 on "Mathematical Modeling, Simulation, and Industrial Applications (M2SI)". David Pardo has received funding from the European Union's Horizon 2020 research and innovation programme under the Marie Sklodowska-Curie grant agreement No 644602, by the RISE Horizon 2020 European Project GEAGAM (644602). Julen Alvarez-Aramberri was also partially funded by the University of the Basque Country UPV/EHU under the grant PIFG05/2011. 


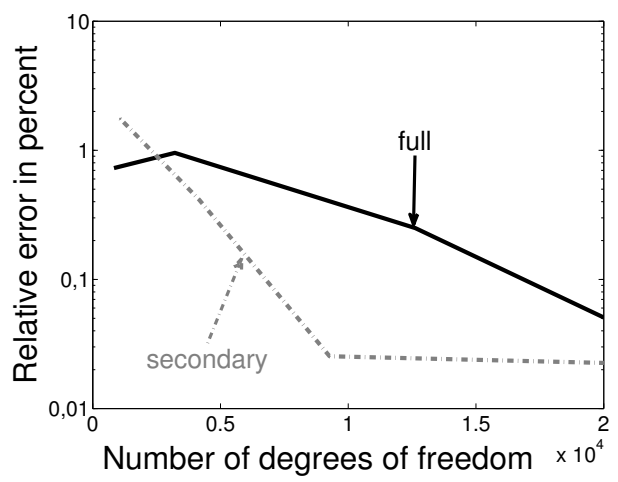

Figure 9: Relative error in logarithmic scale for the apparent resistivity computed with the full and secondary field formulations.

\section{References}

[1] L. Cagniard, Basic theory of the magneto-telluric method of geophysical prospecting, Geophysics 18 (3) (1953) 605-635.

[2] K. Vozoff, The magnetotelluric method in the exploration of sedimentary basins, Geophysics 37 (1) (1972) 98-141.

[3] F. Simpson, K. Bahr, Practical magnetotellurics, Cambridge University Press, 2005.

[4] L. Demkowicz, Computing with hp-Adaptive Finite Elements: Volume 1. One and Two Dimensional Elliptic and Maxwell problems, CRC Press, 2006.

[5] I. Gomez-Revuelto, L. Garcia-Castillo, S. Llorente-Romano, D. Pardo, $3 \mathrm{~d}$ hp-adaptive finite element simulations of bend, step, and magic-T electromagnetic waveguide structures, Journal of Computational Science 5 (2) (2014) $65-75$.

[6] J. Alvarez-Aramberri, D. Pardo, H. Barucq, Inversion of magnetotelluric measurements using multigoal oriented hp-adaptivity, Procedia Computer Science 18 (2013) 1564-1573. 
[7] A. Szymczak, A. Paszyńska, M. Paszyński, D. Pardo, Preventing deadlock during anisotropic $2 \mathrm{~d}$ mesh adaptation in hp-adaptive FEM, Journal of Computational Science 4 (3) (2013) 170-179.

[8] S. Prudhomme, J. Oden, On goal-oriented error estimation for elliptic problems: application to the control of pointwise errors, Computer Methods in Applied Mechanics and Engineering 176 (1) (1999) 313-331.

[9] J. T. Oden, S. Prudhomme, Goal-oriented error estimation and adaptivity for the finite element method, Computers \& Mathematics with Applications 41 (5) (2001) 735-756.

[10] M. Paraschivoiu, A. T. Patera, A hierarchical duality approach to bounds for the outputs of partial differential equations, Computer Methods in Applied Mechanics and Engineering 158 (3) (1998) 389-407.

[11] R. Rannacher, F.-T. Suttmeier, A posteriori error control in finite element methods via duality techniques: Application to perfect plasticity, Computational Mechanics 21 (2) (1998) 123-133.

[12] V. Heuveline, R. Rannacher, Duality-based adaptivity in the hp-finite element method, Journal of Numerical Mathematics JNMA 11 (2) (2003) $95-113$.

[13] P. Šolın, L. Demkowicz, Goal-oriented hp-adaptivity for elliptic problems, Computer Methods in Applied Mechanics and Engineering 193 (6) (2004) 449-468.

[14] D. Pardo, L. Demkowicz, C. Torres-Verdín, L. Tabarovsky, A goaloriented hp-adaptive finite element method with electromagnetic applications. Part i: electrostatics, International Journal for Numerical Methods in Engineering 65 (8) (2006) 1269-1309.

[15] D. Pardo, L. Demkowicz, C. Torres-Verdin, M. Paszynski, A selfadaptive goal-oriented hp-finite element method with electromagnetic applications. Part ii: electrodynamics, Computer methods in Applied Mechanics and Engineering 196 (37) (2007) 3585-3597.

[16] W. Gui, I. Babuška, The h, p and hp versions of the finite element method in 1 dimension. i-iii, Numerische Mathematik 49 (6) (1986) 577683. 
[17] I. Babuška, B. Guo, Approximation properties of the h-p version of the finite element method, Computer Methods in Applied Mechanics and Engineering 133 (3) (1996) 319-346.

[18] C. Schwab, p-and hp-finite element methods: Theory and Applications in Solid and Fluid Mechanics, Clarendon Press Oxford, 1998.

[19] F. Ihlenburg, I. Babuška, Finite element solution of the Helmholtz equation with high wave number part i: The h-version of the FEM, Computers \& Mathematics with Applications 30 (9) (1995) 9-37.

[20] F. Ihlenburg, I. Babuska, Finite element solution of the Helmholtz equation with high wave number part ii: The hp version of the FEM, SIAM Journal on Numerical Analysis 34 (1) (1997) 315-358.

[21] J. Oden, F. Yusheng, Local and pollution error estimation for finite element approximations of elliptic boundary value problems, Journal of Computational and Applied Mathematics 74 (1) (1996) 245-293.

[22] P. Solin, L. Dubcova, J. Cerveny, I. Dolezel, Adaptive hp-FEM with arbitrary-level hanging nodes for Maxwell's equations, Adv. Appl. Math. Mech 2 (4) (2010) 518-532.

[23] R. Hartmann, P. Houston, Goal-oriented a posteriori error estimation for multiple target functionals, Springer, 2003.

[24] R. Hartmann, Multitarget error estimation and adaptivity in aerodynamic flow simulations, SIAM Journal on Scientific Computing 31 (1) (2008) 708-731.

[25] D. Pardo, Multigoal-oriented adaptivity for hp-finite element methods, Procedia Computer Science 1 (1) (2010) 1953-1961.

[26] I. Gomez-Revuelto, L. E. Garcia-Castillo, L. F. Demkowicz, A comparison between pml, infinite elements and an iterative bem as mesh truncation methods for hp self-adaptive procedures in electromagnetics, Progress In Electromagnetics Research 126 (2012) 499-519.

[27] J. Berenger, A perfectly matched layer for the absorption of electromagnetic waves, Journal of Computational Physics 114 (2) (1994) 185-200. 
[28] R. Harrington, J. Harrington, Field computation by moment methods, Oxford University Press, 1996.

[29] W. Chew, W. Weedon, A 3d perfectly matched medium from modified Maxwell's equations with stretched coordinates, Microwave and Optical Technology Letters 7 (13) (1994) 599-604.

[30] F. Teixeira, W. Chew, Analytical derivation of a conformal perfectly matched absorber for electromagnetic waves, Microwave and Optical Technology Letters 17 (4) (1998) 231-236.

[31] F. Teixeira, W. Chew, Pml-fdtd in cylindrical and spherical grids, Microwave and Guided Wave Letters, IEEE 7 (9) (1997) 285-287.

[32] A. Bermúdez, L. Hervella-Nieto, A. Prieto, R. Rodríguez, Perfectly matched layers for time-harmonic second order elliptic problems, Archives of Computational Methods in Engineering 17 (1) (2010) 77107.

[33] P. Joly, An elementary introduction to the construction and the analysis of perfectly matched layers for time domain wave propagation, SeMA Journal 57 (1) (2012) 5-48.

[34] D. Pardo, C. Torres-Verdín, M. Nam, M. Paszynski, V. Calo, Fourier series expansion in a non-orthogonal system of coordinates for the simulation of $3 \mathrm{~d}$ alternating current borehole resistivity measurements, Computer Methods in Applied Mechanics and Engineering 197 (45) (2008) 3836-3849.

[35] D. Pardo, V. Calo, C. Torres-Verdin, M. Nam, Fourier series expansion in a non-orthogonal system of coordinates for the simulation of 3d-dc borehole resistivity measurements, Computer Methods in Applied Mechanics and Engineering 197 (21) (2008) 1906-1925.

[36] A. Ward, J. Pendry, Calculating photonic green's functions using a nonorthogonal finite-difference time-domain method, Physical Review B 58 (11) (1998) 7252.

[37] S. G. Johnson, Notes on perfectly matched layers (pmls), Lecture notes, Massachusetts Institute of Technology, Massachusetts. 
[38] W. C. Chew, Waves and fields in inhomogeneous media, Vol. 522, IEEE press New York, 1995. 